\title{
Elucidation of the Degradation of Poly Aromatic Hydrocarbon (PAH) in Coals During Pyrolysis
}

\author{
Andrew O. Odeh ${ }^{1}$, Samuel E Ogbeide ${ }^{1} \&$ Charity O Okieimen ${ }^{1}$ \\ ${ }^{1}$ Department of Chemical Engineering, University of Benin, Nigeria \\ Correspondence: Andrew O. Odeh, Department of Chemical Engineering, University of Benin, Private Bag 1154, \\ Benin City, Nigeria. Tel: 234-8033-188-797. E-mail: odehandy@yahoo.com
}

Received: October 11, 2016

Accepted: November 14, 2016

Online Published: December 2, 2016

doi:10.5539/eer.v6n2p27

URL: http://dx.doi.org/10.5539/eer.v6n2p27

\begin{abstract}
In this paper, we explore the use of high resolution transmission electron microscopy (HRTEM) in the degradation of the poly aromatic hydrocarbon (PAH) in coals of different ranks subjected to chemical plus heat treatment. The crystallite diameter on peak (10) approximations, La (10), of $37.6 \AA$ for the high rank coal char at $700{ }^{\circ} \mathrm{C}$ fell within the HRTEM's range of minimum-maximum length boundary of $11 \times 11$ aromatic aromatic fringes $(28-44 \AA)$. The $\mathrm{L}_{\mathrm{a}}(10), 30.5 \AA$ for the low rank lignite chars fell nearly on the minimum-maximum length range of $7 \times 7$ aromatic fringes $(17-28 \AA)$.

The HRTEM results showed that the high rank anthracite chars at $700{ }^{\circ} \mathrm{C}$ comprised a higher distribution of larger distribution of larger aromatic fringes (11 x 11 parallelogram catenations). The mechanism for the similarity between coal chars of different ranks was the greater transition occurring in the low rank coals (lignite and sub-bituminous) to match the more resistant medium and high rank coals (bituminous - anthracite). This emphasized that the transitions in the properties of the low rank coals were more thermally accelerated than those of the high rank coals. The total PAHs detected in the coals of different ranks during pyrolysis are dominated by two- and three- ring PAHs. The amount of PAHs increase and then decrease with increase in pyrolysis temperature.
\end{abstract}

Keyword: reactivity, coal-char, HRTEM, chemical properties, poly aromatic hydrocarbons (PAHs)

\section{Introduction}

The increased focus on renewable sources of energy has made current research efforts on coal geared towards clean coal technology. The research efforts on this subject over the past two decades have focused on the chemical treatment of coal (coal demineralization) and of recent on carbon capture and storage (CCS). The determination of the molecular and structural parameters of coal defines the technical performance of coal during coal conversion processes such as gasification, combustion and liquefaction (Odeh, 2015, pp. 129-137; Odeh, 2015, pp. 555-565). The essence of demineralization is to remove or reduce the mineral content in coal as it has been reported that the mineral content in coal melts when coal is subjected to heat treatment during coal conversion processes (Odeh, 2015, pp. 129-137) which results in blocking the carbon active sites thereby reducing the reactivity of the coal and decreasing the emission of pollutants; $\mathrm{NO}_{\mathrm{x}}, \mathrm{SO}_{2}$ and particulates (Odeh, 2015, pp. 2676-2684).

Coal is known to be the most abundant hydrocarbon on earth and its heterogeneous aggregate is made of organic polymeric material with some inorganic impurities. The organic materials are known as macerals, while the inorganic impurities are considered as the minerals (Odeh, 2015, pp. 555-565). When exposed to heat -treatment; the physical, chemical, thermal, mechanical and electrical properties of coal undergo transformation (Odeh, 2015, pp. 129-137). The chemical transformation involves the change in the organic chemical structure while the physical transformation involves a change in the char morphology and porosity (Odeh, 2015, pp. 2676-2684). The heterogeneous and complex nature of coal has made studies in understanding coal as a resource material an on-going research exercise in different laboratories around the world with the use of advanced analytical techniques such as FTIR, XRD, HRTEM, SAXS and ${ }^{~} \mathrm{NMR}$.

HRTEM has proven to be the only technique available for observing the molecular structure of coal and coal chars (Mathews, Jones, Pappano, Hurt, \& Schobert, 2011; Van Niekerk, 2008) and recent developments by 
Mathews et al. (2011) have enabled investigators to assign aromatic sizes to the extracted HRTEM images (Mathews, Jones, Pappano, Hurt, \& Schobert, 2011). In this method the aromatic fringes are represented by parallelogram sheets of different sizes (Mathews, Jones, Pappano, Hurt, \& Schobert, 2011; Van Niekerk, 2008). Although not truly an indication of the large molecular moieties in coal, this approach provides a reasonable starting point for qualitatively describing the diversity and distribution of the aromatic rafts present in coal (Van Niekerk, 2008). Other investigators such as (Solum, Pugmire, \& Grant, 1989; Solum, Sarofim, Pugmire, Fletcher, \& Zhang, 2001) have, however, represented the aromatic rafts as either linear or circular catenations during lattice calculations.

In this work, the high resolution thermal electron microscope (HRTEM) was applied to the characterization and quantitative structural study of six coals of different rank with emphasis on the degradation of poly aromatic hydrocarbons (PAH) in coals. PAHs are ubiquitous environmental pollutants generated primarily during the incomplete combustion of carbonaceous materials such as coal, oil and wood (Liu, Xie, Zhao, Pan, \& Riley, 2000; Wang, Yousaf, Sun, Zhang, Zhang, \& Liu, 2016). PAHs are grouped according to three sources of origin: petrogenic (Petroluem based); pyrogenic (coal and biomass burning based) and natural (Liu, Xie, Zhao, Pan, \& Riley, 2000). PAHs are hydrocarbons or organic materials containing only carbon and hydrogen (Zhou, Jin, Xiao, Zhong, \& Huang, 2009); that are composed of multiple aromatic rings (Pergal, Tesic, \& Popovic, 2013). Based on ths definition, benzene that have one aromatic ring is not considered as a PAH but compounds such as naphthalene (two aromatic rings); anthracene and phenanthrene (three aromatic rings) and other multiple aromatic rings are poly aromatic hydrocarbons with no appendages of halogens or other substituent compounds (Hsu, Liu, Hung, Chang, \& Chang, 2016).They are lipophilic in nature and insoluble in water (Wang, Yousaf, Sun, Zhang, Zhang, \& Liu, 2016). The emmission of these polycyclic aromatic matter into the atmosphere has its environmental (Alves, Vincente, Gomes, Nunes, Duarte, \& Bandowe, 2016) and health effects (Abdel-Shafy, \& Mansour, 2016). For instance, daily exposures to particulate matter associated with PAHs will lead to inceased incidences of premature death, chronic asthma as well as other air borne diseases both in adult and children (Ravindra, Sokhi, \& Grieken, 2008; Irha, Reinik, Jefimova, Koroljova, Raado, Hain, Uibu, \& Kuusik, 2015).

It is envisaged that the use of advanced chemical engineering technique such as the application of HRTEM would help to reveal and identify the dinstinct types of PAHs in coal and coal chars during pyrolysis. To achieve this objective, all the coal samples used were demineralized to account for only the organic component of coal.

\section{Experimental}

\subsection{Sample Preparation}

Six Southern Hemisphere coal samples of varying rank were used: a lignite coal from Germany (LIG); A sub-bituminous coal from Nigeria (SUB); two South African bituminous coals (one is low volatile bituminous (BIT-LV) and the other, high volatile bituminous coal (BIT-HV); South African semi-anthracite (SA); and anthracite from South Africa (ANT). The coal samples were pulverized to coal particle size of $\leq 75 \mu \mathrm{m}$ by employing a mechanical size reduction jaw crusher (Samuel Osborne (SA) LTD, model: 66YROLL) and a Fritsch P-14 rotary mill containing ceramic balls (Model number: $46-126$ ). The required particle size of $-75 \mu \mathrm{m}$ was finally obtained from screening the particles from the rotary mill using a $75 \mu \mathrm{m}$ screen. All the samples were stored under argon in sealed bags.

The prepared coal samples were demineralized by sequential leaching with hydrofluoric acid (HF) and hydrochloric acid $(\mathrm{HCl})$ as detailed in odeh (Odeh, 2015, pp. 2676-2684). The $\mathrm{HF}(48 \%)$ and $\mathrm{HCl}(32 \%)$ were obtained from Associated Chemical Enterprise (ACE), South Africa.

\subsection{Apparatus and Procedure}

The coal samples $(40 \mathrm{~g})$ were placed in a ceramic boat in a horizontal tube furnace at atmospheric conditions initially. The samples were flushed with nitrogen (AFROX, ultra high purity grade) at atmospheric conditions, to remove oxygen from the oven for $15 \mathrm{~min}$ at a flow rate of $1 \mathrm{~L} / \mathrm{min}$. The furnace was then heated at $25^{\circ} \mathrm{C} / \mathrm{min}$ to the target temperature, and held isothermally for $60 \mathrm{~min}$. The target temperature ranged from 450 to $700{ }^{\circ} \mathrm{C}$ at 50 ${ }^{\circ} \mathrm{C}$ interval, while keeping the samples under a nitrogen atmosphere. The char samples were stored in sealed bags.

The coal samples of $-75 \mu \mathrm{m}$ were hand-ground for 15 mins with a pestle and mortar in order to produce an ultrafine powdered sample. The finely ground samples were subjected to image acquisition using a Tecnai F20 FEGTEM and photographed using a Gatan CCD camera (Model 895) from where fringes (thin layers of particles that were situated at least partially across the holes in the carbon film) were obtained. The acquired images were converted to tif format for lattice fringe analysis with the use of image processing toolkit of Photoshop and 
Material studio and as detailed in (Mathews, Jones, Pappano, Hurt, \& Schobert, 2011; Van Niekerk, 2008).

\section{Results and Discussion}

HRTEM images of the old rank coals are shown in Fig.1. Visual inspection of multiple micrographs of the six coals shows that anthracite coal had a less oriented structure in comparison with the other five coals. These observations tend to be consistent with what was observed for the pore size distribution and aromaticity values obtained from SEM (Scanning electron microscope) and XRD (X-ray Diffractogram) analyses and as reported in odeh (Odeh, 2015, pp. 129-137). It is therefore clear that coal ANT contained fewer poly-condensed molecular units, with a less ordered structure.

The HRTEM micrographs of each coal were processed according to the method discussed in 2.2. An example of the obtained processed images from the cropping step to the binary conversion step, through to the final lattice-fringe extracted image is illustrated for coal BIT-LV in Fig. 2. From the Figure it is clear that the skeletonised image provides a good representation of the presence and orientation of the different fringes or aromatic rafts (Mathews, Jones, Pappano, Hurt, \& Schobert, 2011). The image processing toolkit of Reindeer Graphics was further applied to determine the length (in Angstroms) of each extracted aromatic fringe. A typical size-distribution (where the fringes are default coloured according to size) is presented in a fringe-extracted HRTEM image of coal ANT as illustrated in Fig.3. Similar results were obtained for the other coal samples used in this study.

Previous studies by Mathews et al. (2011) and Van Niekerk (2008) reported results that are similar to the resuts obtained in this work.. In this method the aromatic fringes are represented by parallelogram sheets of different sizes (Mathews, Jones, Pappano, Hurt, \& Schobert, 2011; Van Niekerk, 2008). Although not truly an indication of the large molecular moieties in coal, this approach provides a reasonable starting point for qualitatively describing the diversity and distribution of the aromatic rafts present in coal (Van Niekerk, 2008). As an example, all fringes exhibiting sizes between $4.4 \AA$ and $5.9 \AA$ were assigned to the anthracene/phenanthrene catenation, while fringes between $5.9 \AA$ and $9.9 \AA$ were assigned to the $2 \times 2$ parallelogram catenation etc. Fringes with lengths smaller than $3 \AA$ were assigned to benzene although this could have included some noise that was not totally removed during the filtering process. The obtained fringes were therefore classified within the different parallelogram catenation groups and the relative frequency of each specific parallelogram raft size was expressed as a percentage of the total amount of fringes. For each cropped micrograph of each coal more than 4000 fringes were extracted and subjected to image analyses and aromatic raft size determination. The obtained aromatic raft size distributions with respect to fringe length for all the coals samples exhibited similar trend as illustrated in Fig. 4 and 5. The aromatic raft size distributions of all six coals exhibited similar Lorentzian type behaviour, which is consistent with what was observed by other researchers for bituminous coals and as reported in (Van Niekerk, 2008). All six coals exhibit a greater abundance of fringes concentrated in the $2 \times 2$, $3 \times 3$, and $4 \times 4$ region, while the abundance of larger fringes decrease with increasing fringe length. The higher rank coals are surprisingly similar in their distribution of aromatic fringes in the range extending from Benzene to $5 \times 5$. Coal ANT, however, show a slightly higher abundance of aromatic fringes in the range greater than $5 \times 5$, when compared to the other five coals. All six coals exhibit a greater abundance of fringes concentrated in the $2 \times 2,3 \times 3$, and $4 \times 4$ region, while the abundance of larger fringes decrease with increasing fringe length. The two low rank coals (GER and NGR) are surprisingly similar in their distribution of aromatic fringes in the range extending from Benzene to $6 \times 6$. Similar trend in the distribution of aromatic fringes was demonstrated by the two medium rank coals (BIT-LV and BIT-HV) while the two high rank coals (SA and ANT) exhibited similar trend. This could be attributed to the fact the coals of the same rank tends to exhibit similar devolatilization mechanism during pyrolysis (Odeh, 2015, pp. 555-565).

In addition, it could be observed that the aromatic rafts confined to the benzene to $3 \times 3$ fringe lengths were more abundant in the low rank coals. The observations are consistent with what was determined through another analytical and also reported by (Liu, Xie, Zhao, Pan, \& Riley, 2000). This confirms that the low rank coals are least aromatic (Odeh, 2015, pp. 129-137; Wang, Yousaf, Sun, Zhang, Zhang, \& Liu, 2016) and poly-condensed while the inverse is true for coal high rank coals of SA and ANT.

From Fig.6, one could observe the variation in the distribution of the poly-aromatics in the different coal samples used in the study. Fig. 6 demonstrates the occurrence as well as the spead of these aromatics. These spread can be described as random, irregular aand dramatic in nature potraying the heterogeneous and complex nature of coal. Definite trend could not be observed for the distribution of these aromatics in coal wth an increase in values then a decrease. However, the results obtained from this study gives an understanding of the distribution of poly-aromatic hydrocarbons in coal during pyrolysis and would be a useful and meaningful tool for reaseachers 
that are into molecular modelling of coal and coal associated products. The results revealed that napthalene and anthracene were more predominant in the coal and coal char material than the other numerous PAHs (Bartonova, 2015).These aromatics belongs to the group that classified as "small" (PAHs containing two to four fused aromatic ring) molecular weight poly aromatic hydrocarbons (PAHs) (Ravindra, Sokhi, \& Grieken, 2008; Bartonova, 2015). PAHs are ubiquitous environmental pollutants generated primarily during the burning of carbonaceous materials such as coal (Abdel-Shafy, \& Mansour, 2016).The PAHs generated from coal utilization processes are regarded as pyrogenic PAHs (Wen, Yang, Zeng, \& Huang, 2016). They are toxic, mutagenic and carcinogenic in nature (Shin, Kim, Jeon, Kang, Jeong, Yeon, \& Somasundaram, 2013). PAHs generally are soluble, and of high melting and boiling points and exhibits characteristic UV(Ultra violet) absorbance spectra (Ravindra, Sokhi, \& Grieken, 2008). Each ring structure has a unique UV spectrum when examined under a light microscope (Wen, Yang, Zeng, \& Huang, 2016). The use of high resolution thermal electron microscope (HRTEM), has helped to reveal and identify dinstinct sources of PAH during pyrolysis of coal of different ranks. As can be seen in Fig. 6, benzene (which is not a PAH, since it has only one fused ring), naphthalene, and anthracene are the only PAHs identified in this study. Though phenanthrene is three-ringed PAH, it was not deteted in this study. Fig.6 demonstrated that PAH formation is temperature dependent and their distribution pattern is governed by both thermodynamic and kinetic factors of the process (Abdel-Shafy, \& Mansour, 2016), specifically, as in pyrolysis in this investigation.

A parameter that is used to test the level of pollution of PAHs in an environment and to differentiate between pyrogenic and petrogenic PAHs is the ratio of phenanthrene (PO) to anthracene (AO) (Abdel-Shafy, \& Mansour, 2016). However, in this investigation, it was observed that phenanthrene was either not detected nor does not exist in the pyrolysis zone of a typical gasifier fed with demineralized coal samples. Combustion processes has been assigned a value of $\mathrm{PO} / \mathrm{AO}$ of less than $5(\mathrm{PO} / \mathrm{AO}<5)$ (Wen, Yang, Zeng, \& Huang, 2016). For the purpose of understanding the degradation of PAHs in coal and because of the little or no value of phenanthrene in the coal samples used in this work, the ratio of anthracene to naphthalene was used as the pollution matrix parameter. As can be seen in Table 1, the pollution parameter ratio do not have a regular trend but it was observed that the value of this ratio decreases with increasing pyrolysis temperature after the climax temperature of $600{ }^{\circ} \mathrm{C}$. This observation cut across all the coal samples. There is likely the possibility of this ratio dropping further with increasing pyrolysis temperature which indicates that the rate of coal PAH pollution contamination to an environment decreases with increasing pyrolysis temperature. Similarly, the crystallite diameter of the coal samples exhibited different devolatilization mechanisms as reflected and presented in Table 1. The two low rank coals (LIG and SUB) had values of crystallire diameter that are close (30.8 for LIG and 30.7 for SUB). The same trend was observed for the two medium rank (33.7 for BIT-LIV and 33.6 BIT-HV) and the two high rank coals also demonstrated similar trend (37.6 and 37.4). The data obtained from this study can be standardized and use for comparing and evaluating PAHs concentrations in a contaminated area in coal processing plant.

\section{Concluding Remarks}

The advanced characterization conducted on the six coal samples revealed the respective molecular and structural nature of each coal and its subsequent coal chars. HRTEM analyses showed a similar Lorentzian-type behaviour as observed other analytical techniques. From the HRTEM analyses it was evident and as expected that coal ANT contained larger amounts of aromatic fringes (average size range of $3 \times 3$ ) confined to higher molecular masses. The aromatic fringes demonstrated the distribution of inherent poly aromatic hydrocarbon in coal and coal chars. The total PAHs detected in the coals of different ranks during pyrolysis are dominated by two- and three- ring PAHs. The amount of PAHs increase and then decrease with increase in pyrolysis temperature.

Low molecular weight poly aromatic hydrocarbon of naphthalene and anthracene were identified as the predominant PAHs in coal during pyrolysis irrespective of the petrographic composition of the parent coal. PAHs of this class has been identified to be souble in water making them more available for biologically update and degradation and easily bound to particulate air pollution, soils and sediments. Daily exposure to these PAHs leads to adverse health concerns as these PAHs has be classified as cancigenic and mutagenic. The understanding obtained from this study would help coal scientists and all working in coal processing plants the temperature to limit their exposure to in the course of processing and utilization of coal and coal allied products. 


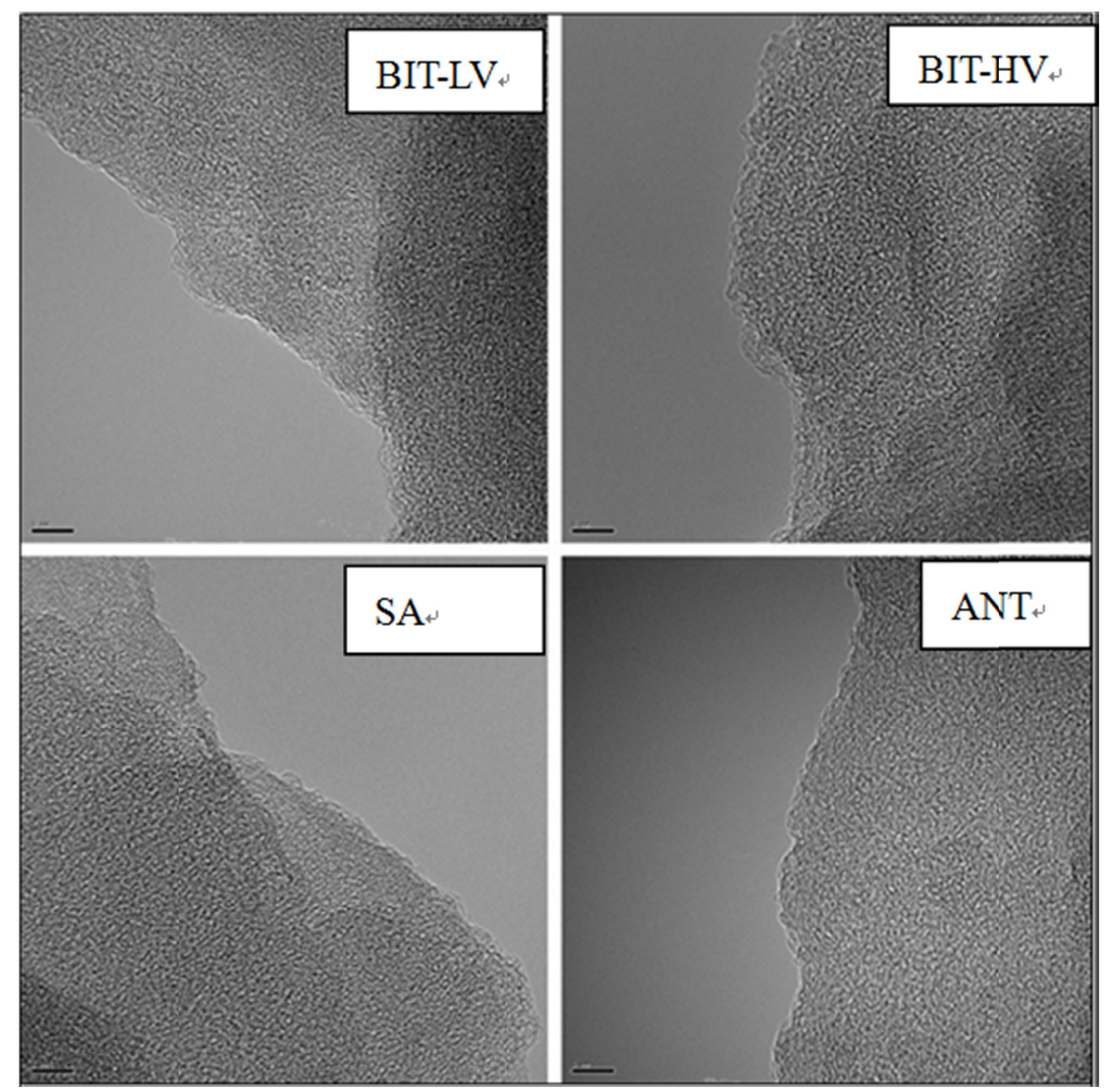

Figure 1. HRTEM micrographs for coal chars
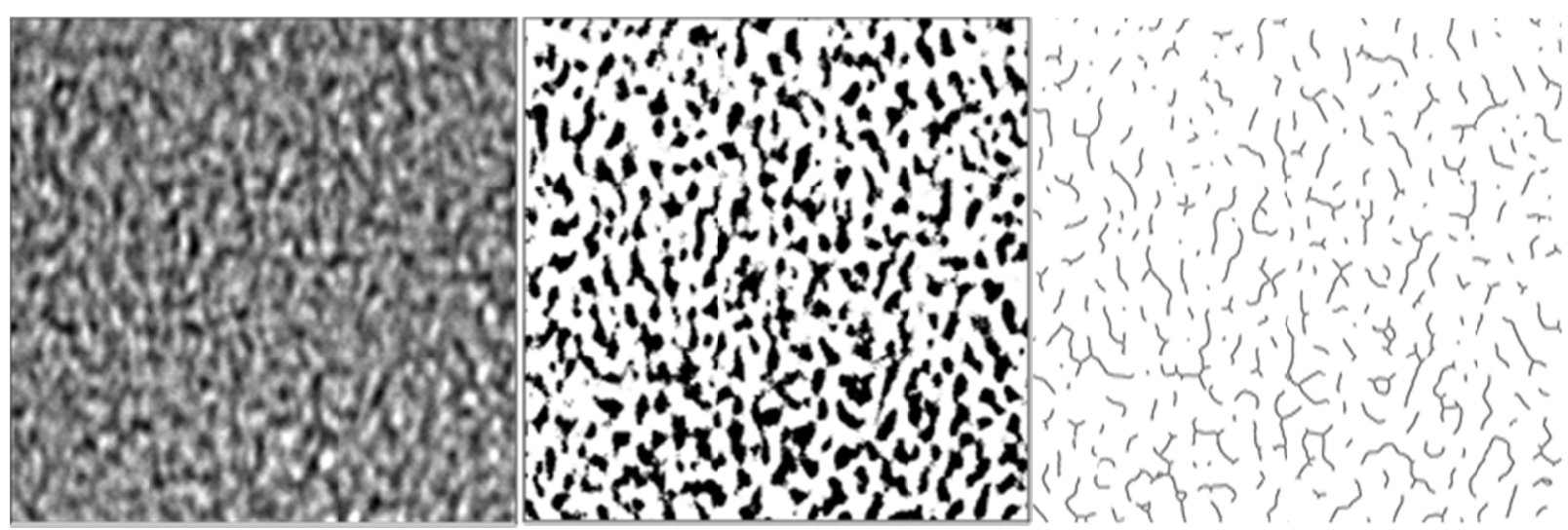

Figure 2. From left to right: Cropped HRTEM image; FFT and thresholded image; and skeletonised and lattice fringe image 


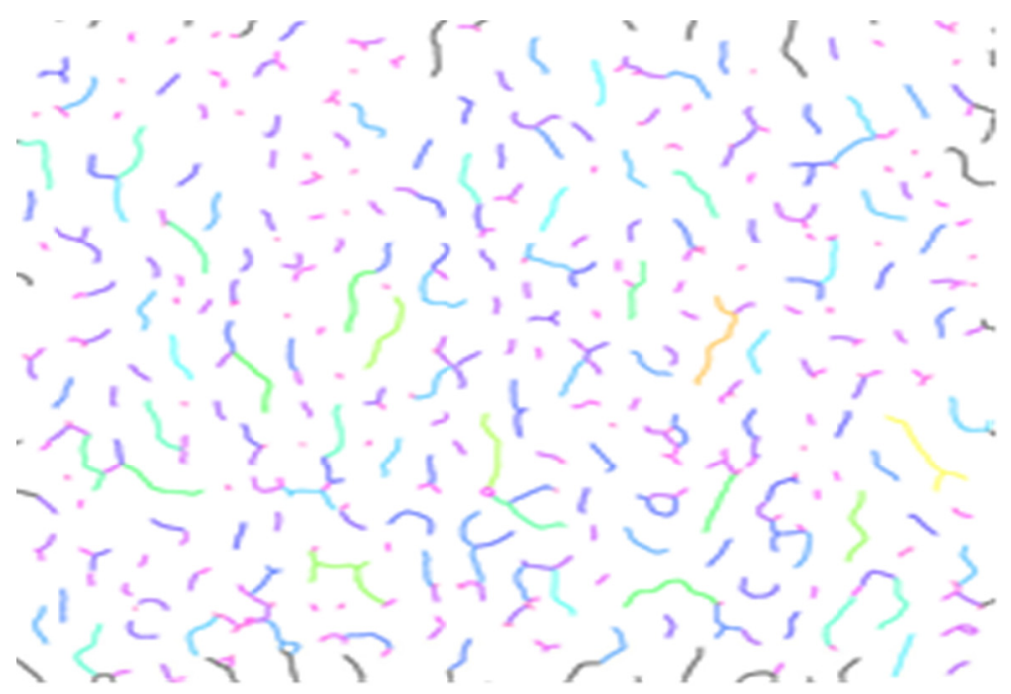

Figure 3. Fringe length coloured according to sizes

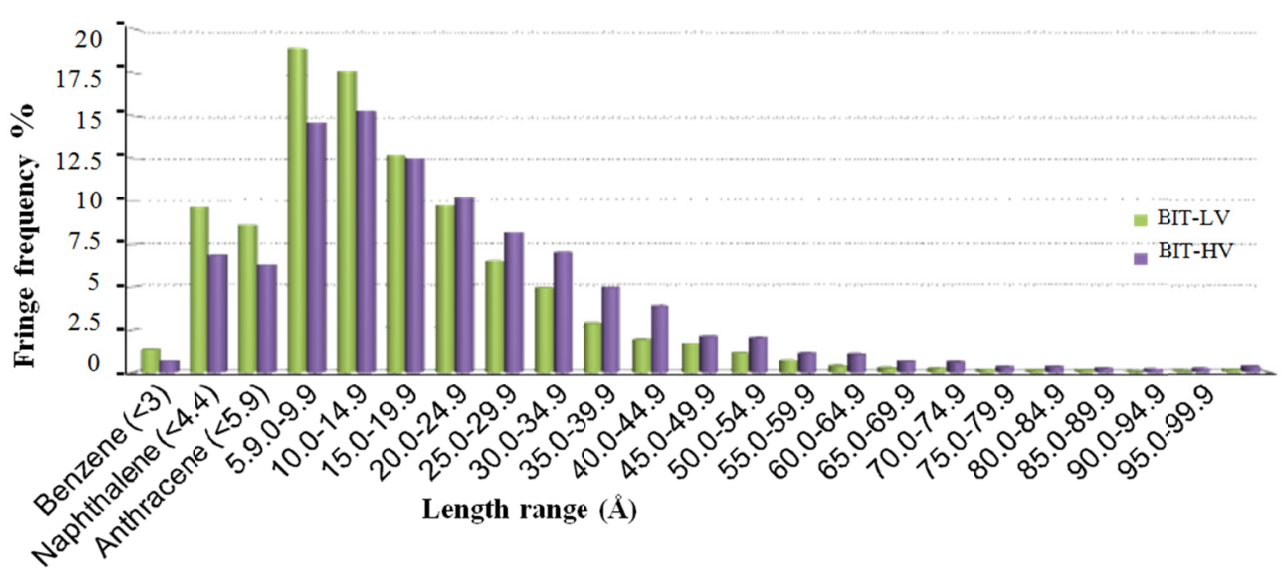

Figure 4. Aromatic raft size distribution w.r.t to fringe length for coals BIT-LV

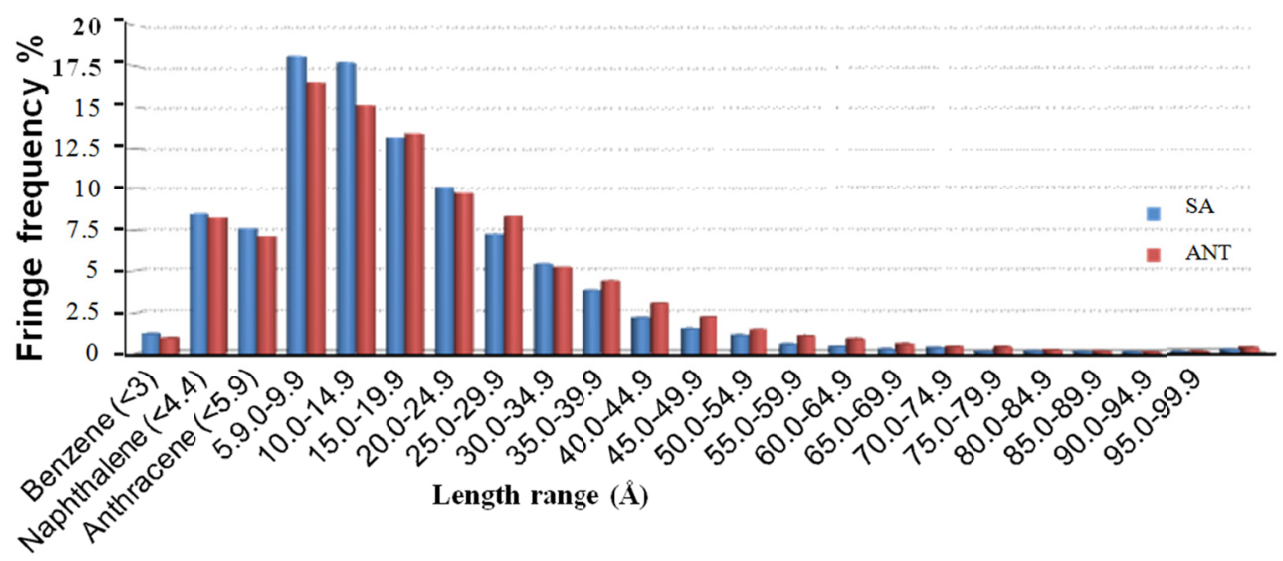

Figure 5. Aromatic raft size distribution w.r.t to fringe length for coals SA 


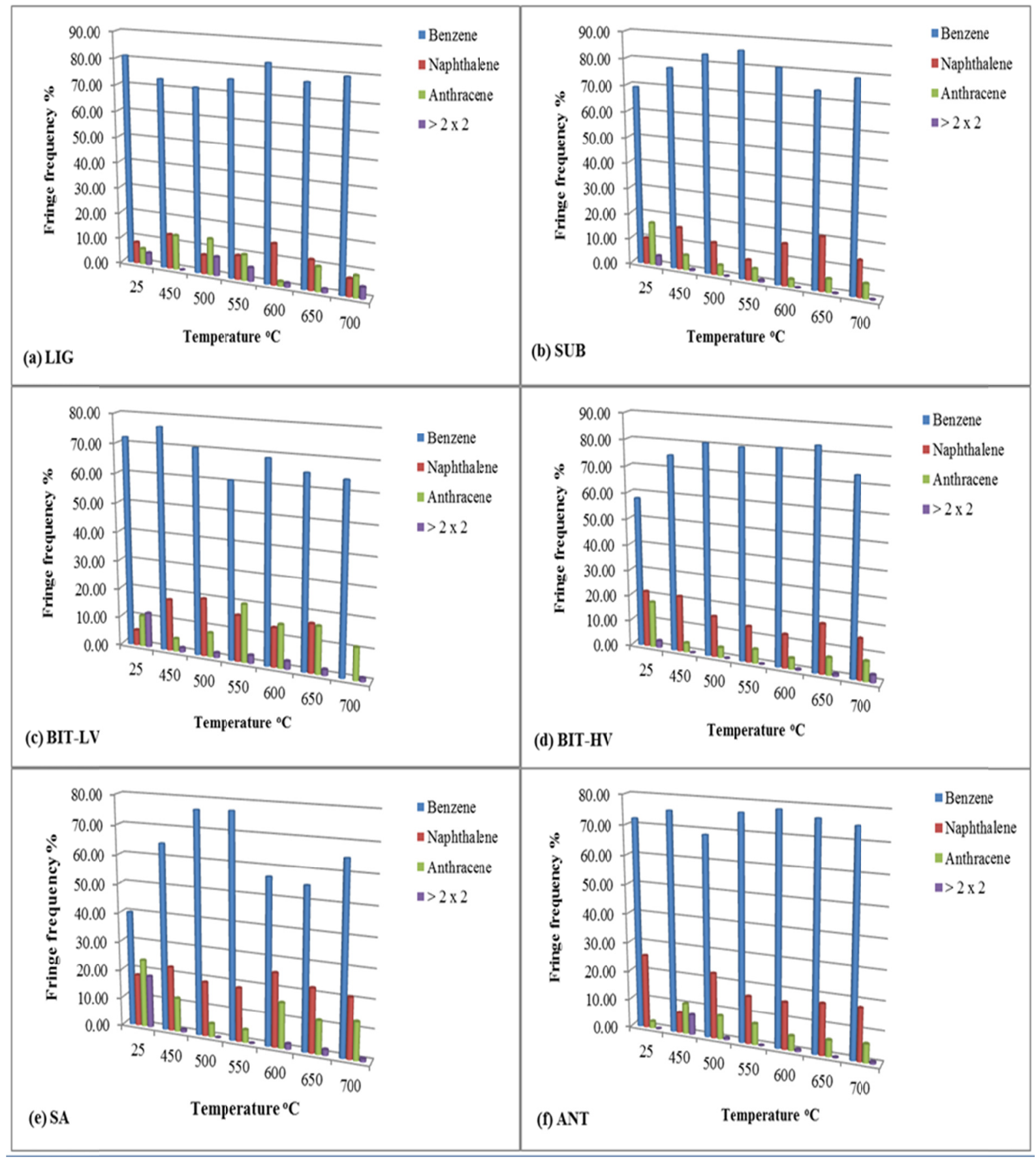

Figure 6. Distribution of fringes of Poly-aromatic hydrocarbon during pyrolysis 
Table 1. Determined chemical properties of heat-treated coal (chars)

\begin{tabular}{|l|l|l|l|l|l|l|}
\hline \multirow{2}{*}{ CHAR } & \multicolumn{6}{|c|}{ LIGNITE (LIG) } \\
\cline { 2 - 8 } & $\mathbf{4 5 0}$ & $\mathbf{5 0 0}$ & $\mathbf{5 5 0}$ & $\mathbf{6 0 0}$ & $\mathbf{6 5 0}$ & $\mathbf{7 0 0}$ \\
\hline Crystallite diameter $\left(\mathrm{L}_{\mathrm{a}}\right) \AA$ & $30.4 \pm 0.2$ & $30.6 \pm 0.1$ & $30.5 \pm 0.3$ & $30.8 \pm 0.2$ & $30.6 \pm 0.1$ & $30.8 \pm 0.3$ \\
\hline AO/NO ratio & 1.0 & 0.5 & 0.9 & 8.8 & 1.2 & 0.8 \\
\hline & \multicolumn{7}{|c|}{ SUB-BITUMINOUS (SUB) } \\
\hline Crystallite diameter $\left(\mathrm{L}_{\mathrm{a}}\right) \AA$ & $30.6 \pm 0.1$ & $30.5 \pm 0.3$ & 30.7 & $30.6 \pm 0.3$ & $30.7 \pm 0.1$ & $30.7 \pm 0.2$ \\
\hline AO/NO ratio & 2.8 & 3.1 & 1.6 & 5.4 & 3.9 & 2.4 \\
\hline & \multicolumn{7}{|c|}{ BITUMINOUS (BIT-LV) } \\
\hline Crystallite diameter $\left(\mathrm{L}_{\mathrm{a}}\right) \AA$ & $33.6 \pm 0.1$ & $33.5 \pm 0.3$ & $33.6 \pm 0.2$ & $33.8 \pm 0.2$ & $33.6 \pm 0.3$ & $33.7 \pm 0.2$ \\
\hline AO/NO ratio & 0.2 & 0.4 & 1.1 & 1.3 & 0.9 & 0.5 \\
\hline & \multicolumn{7}{|c|}{ BITUMINOUS (BIT-HV) } \\
\hline Crystallite diameter $\left(\mathrm{L}_{\mathrm{a}}\right) \AA$ & $32.9 \pm 0.3$ & $33.5 \pm 0.2$ & $33.6 \pm 0.3$ & $33.5 \pm 0.2$ & $33.4 \pm 0.3$ & $33.6 \pm 0.3$ \\
\hline AO/NO ratio & 6.1 & 4.0 & 2.6 & 3.0 & 2.8 & 2.1 \\
\hline & \multicolumn{7}{|c|}{ SEMI-ANTHRACITE (SA) } \\
\hline Crystallite diameter $\left(\mathrm{L}_{\mathrm{a}}\right) \AA$ & $36.9 \pm 0.2$ & $37.4 \pm 0.1$ & $37.6 \pm 0.3$ & $37.5 \pm 0.1$ & $37.3 \pm 0.3$ & $37.6 \pm 0.2$ \\
\hline AO/NO ratio & 1.9 & 4.1 & 1.9 & 4.3 & 1.6 & 1.6 \\
\hline & \multicolumn{7}{|c|}{ ANTHRACITE (ANT) } \\
\hline Crystallite diameter $\left(\mathrm{L}_{\mathrm{a}}\right) \AA$ & $37.4 \pm 0.2$ & $37.6 \pm 0.1$ & $37.5 \pm 0.1$ & $37.6 \pm 0.2$ & $37.5 \pm 0.2$ & $37.4 \pm 0.3$ \\
\hline AO/NO ratio & 0.7 & 2.8 & 2.3 & 3.2 & 3.1 & 2.9 \\
\hline
\end{tabular}

AO - Anthracite ; NO - Napthalene

\section{References}

Abdel-Shafy, H. I., \& Mansour, M. S. M. (2016). A review on polycyclic aromatic hydrocarbons: Source, environmental impact, effect on human health and remediation. Egyptian Journal of Petroleum, 25, 107-123.

Alves, C. A., Vincente, A. M. P., Gomes, J., Nunes, T., Duarte, M., \& Bandowe, B. A. M. (2016). Polycyclic aromatic hydrocarbons (PAHs) and their derivatives ( Oxygenated - PAHs, nitrated - PAHs and azaarenes) in size fractionated particles emitted in an urban road tunnel. Atmospheric Research, 180, 128-137.

Bartonova, L. (2015). Unburned carbon from coal combustion ash: An overview. Fuel Processing Technology, 134, 136-158.

Hsu, W. T., Liu, M. C., Hung, P. C., Chang, S. H., \& Chang, M. B. (2016). PAH emissions from coal combustion and waste incineration. Journal of Hazardous Materials, 318, 32-40.

Irha, N., Reinik, J., Jefimova, J., Koroljova, A., Raado, L. M., Hain, T., Uibu, M., \& Kuusik, R. (2015). PAHs in leachates from thermal power plant wastes and ash-based construction materials. Environmental Science and Pollution Research, 22, 11877-11889.

Liu, K., Xie, W., Zhao, Z-B., Pan, W-B., \& Riley, J. T. (2000). Investigation of poly aromatic hydrocarbons in fly ash from fluidized combustion systems. Environmental Science Technology, 34, 2273-2279.

Mathews, J. P., Jones, A. D., Pappano, P. J., Hurt, R., \& Schobert, H. H. (2011). New insights into coal structure from the combination of HRTEM and laser desorption ionization mass spectrometry. In Proceedings of the 11th International Conference on Coal Science held in San Francisco, CA, 2001. San Francisco, CA.

Odeh, A. (2015). Comparative study of the aromaticity of the coal structure during the char formation process under both conventional and advanced analytical techniques. Energy \& Fuels, 29, 2676-2684.

Odeh, A. (2015). Exploring the potential of petrographics in understanding coal pyrolysis. Energy, 87, 555-565.

Odeh, A. (2015). Quatitative ATR-FTIR analysis and its application to coal char of different ranks. Journal of Fuel Chemistry and Technology, 43, 129-137.

Pergal, M. M., Tesic, Z. L., \& Popovic, A. R. (2013). Poly aromatic hydrocarbons: Temperature driven formation and behavior during coal combustion in a coal - fired power plant. Energy \& Fuel, 27, 6273-6278.

Ravindra, K., Sokhi, R., \& Grieken, R. V. (2008). Atmospheric polycyclic aromatic hydrocarbons: Source attribution, emission factors and regulation. Atmospheric Environment, 42, 2895-2921.

Shin, S. K., Kim, W. L., Jeon, T. W., Kang, Y. Y., Jeong, S. K., Yeon, J. M., \& Somasundaram, S. (2013). Hazardous waste characterization among various thermal processes in South Korea: A comparative analysis. Journal of Hazardous Materials, 260, 157-166. 
Solum, M. S., Pugmire, R. J., \& Grant, D. M. (1989). Carbon - 13 solid state NMR of Argonne - premium coals. Energy \& Fuels, 3, 187-193.

Solum, M. S., Sarofim, A. F., Pugmire, R. J., Fletcher, T. H., \& Zhang, H. (2001). 13C NMR analysis of soot produced from model compounds and a coal. Energy \& Fuels, 15, 961-971.

Van Niekerk, D. (2008). Structural elucidation, molecular representation and solvent interactions of vitrinite-rich and inertinite-rich South African coals. Pennsylvania: Pennsylvania State University. USA. (Thesis-PhD), 195p.

Verma, S. K., Masto, R. E., Gautam, S., Choudhury, D. P., Ram, L. C., Maiti, S. K., \& Maity, S. (2015). Investigations on PAHs and trace elements in coals and its combustion residues from a power plant. Fuel, 162, 138-147.

Wang, R., Yousaf, B., Sun, R., Zhang, H., Zhang, J., \& Liu, G. (2016). Emission characterization and ${ }^{{ }^{13}} \mathrm{C}$ values of parent PAHs and nitro- PAHs in size - segregated particulate matters from coal - fired plants. Journal of Hazardous Materials, 318, 487-496.

Wen, X., Yang, Y., Zeng, F., \& Huang, D. (2016). Influence of temperature and airflow on polycyclic aromatic hydrocarbons (PAHs) by simulated self-combustion of coal partings. Journal of Environmental Chemical Engineering, 4, 3601-3609.

Zhou, H., Jin, B., Xiao, R., Zhong, Z., \& Huang, Y. (2009). Distribution of poly aromatic hydrocarbons in fly ash during coal and residual char combustion in a pressurized fluidized bed. Energy \& Fuel, 23, 2031-2034.

\section{Copyrights}

Copyright for this article is retained by the author(s), with first publication rights granted to the journal.

This is an open-access article distributed under the terms and conditions of the Creative Commons Attribution license (http://creativecommons.org/licenses/by/3.0/). 